\title{
Spin dependent fragmentation functions at Belle
}

\author{
R. $\operatorname{Seidl}\left({ }^{1}\right)$, M. Leitgab $\left({ }^{2}\right)$, A. $\operatorname{Vossen}\left({ }^{3}\right)$, F. $\operatorname{Giordano}\left({ }^{2}\right)$, N. $\operatorname{Kobayashi}\left({ }^{4}\right)$, \\ M. Grosse-Perdekamp $\left({ }^{2}\right)$, A. Ogawa $\left({ }^{5}\right)\left({ }^{6}\right)$, C. $\operatorname{Hulse}\left({ }^{7}\right)$ and G. Schnell $\left({ }^{7}\right)\left({ }^{8}\right)$ \\ for the Belle Collaboration \\ $\left({ }^{1}\right)$ RIKEN - Japan \\ (2) University of Illinois - Urbana, IL, USA \\ $\left(^{3}\right)$ University of Indiana - Bloomington, ID, USA \\ $\left({ }^{4}\right)$ Tokyo Institute of Technology - Japan \\ $\left({ }^{5}\right)$ Brookhaven National Laboratory, Upton, NY, USA \\ $\left({ }^{6}\right)$ RIKEN BNL Research Center - Upton, NY, USA \\ $\left({ }^{7}\right)$ University of the Basque Country - Bilbao, Spain \\ $\left.{ }^{8}\right)$ Ikerbasque - Spain
}

ricevuto il 18 Aprile 2013

Summary. - The Belle detector at the $\mathrm{KEKB} e^{+} e^{-}$collider provides large amounts of statistics to study the fragmentation of light quarks into final state hadrons. In addition to unpolarized fragmentation functions also spin dependent fragmentation can be studied. Belle has successfully extracted asymmetries related to the Collins and interference fagmentation functions for charged pions.

PACS 13.87.Fh - Fragmentation into hadrons.

PACS 13.88.+e - Polarization in interactions and scattering.

\section{1. - Introduction}

There are three leading twist parton distribution functions (PDFs) in the nucleon. The unpolarized PDF and the helicity distribution function have been measured to some precision predominantly in deep inelastic scattering and hadron hadron collisions. Smaller- $x$ regions have not been covered too much so far and need a polarized electron ion collider in the future. The third leading PDF is the transversity distribution which did not get measured at all until very recently and only with the help of spin dependent fragmentation functions. Unlike the two other distribution functions, transversity is a chiral-odd quantity and it can therefore be only measured together with another chiralodd quantity. That second chiral-odd quantity could be either an antiquark transversity distribution in the Drell-Yan process or a chiral-odd fragmentation function such as the Collins or Interference fragmentation function. As a consequence, it is of great importance to cleanly measure those fragmentation functions. In the $e^{+} e^{-}$annihilation process, one mostly produces a quark-antiquark pair which could be transversely polarized. Using the combination of two chiral-odd fragmentation functions for both hemispheres one is able to only extract asymmetries related to those fragmentation functions. 


\section{2. - The Belle experiment}

The Belle [1] experiment is located at the asymmetric $3.5 \mathrm{GeV}$ on $8.0 \mathrm{GeV} e^{+} e^{-}$ collider KEKB [2]. The main purpose of this nearly hermetic detector is the study of CP violation and the CKM matrix in the production ob $B$ mesons off the $\Upsilon(4 S)$ resonance at $\sqrt{s}=10.58 \mathrm{GeV}$. However, substantial data is taken also $60 \mathrm{MeV}$ below where only light and charm quark pairs are initially produced. Belle consists of a silicon vertex detector for intial vertexing, a central drift chamber for particle tracking, Time-of-flight and Aerogel detectors for hadron identification and an electromagentic Calorimeter within a Solenoid of $1.5 \mathrm{~T}$ field strength. The return yoke is instrumeted with Glass RPCs to identify muons and $K_{L}$. The combination of the particle identification detectors and the energy loss measurements in the drift chambers allow for good pion and kaon identification throught most of the kinematic reach.

\section{3. - Collins}

The Collins fragmentation function was first suggested by Collins [3] to describe the large single transverse spin asymmetries seen in the E704 experiment [4]. It describes the fragmentation of transversely polarized quarks into an observable azimuthal asymmetry in the yield of final state hadrons around the quark's direction. In $e^{+} e^{-}$annihilation one therefore needs to find the quarks direction which is experimentally approximated by the thrust axis. One then detects hadron pairs in opposite hemispheres and measures their anlges with respect to the production plane defined by the lepton axis and the thrust axis. The cosine of the sum of these two angles is then proportional to the product of the Collins fragmentation functions for the quark and the antiquark side divided by the corresponding unpolarized fragmentation functions. As other processes than quarkantiquark production are present several event level selection criteria are necessary. In order to remove $B$ meson events also the thrust value needs to be above 0.8 and in order to reduce $\tau$ pair events a minimum visible energy of $7 \mathrm{GeV}$ is selected. One selects pairs of charged pions which need to be in opposing hemispheres as defined by the thrust axis. In the Belle experiment we require the hadrons and the thrust axis to be well within the barrel region of the detector in order to minimize acceptance effects. However, some acceptance effects remain. Those were eliminated by not directly calculating azimuthal asymmetries of charged pion pairs, but rather building ratios of normalized yields between pion pairs of same and opposite charge. Afterwards the cosine modulation is fit. The results, first published for a small data sample below the $\Upsilon(4 S)$ resonance [5] and later for a larger data set [6] show substantial asymmetries which are increasing with fractional energies of the two hadrons as can be seen in fig. 1. These results have been applied together together with the semi-inclusive DIS data from HERMES [7] and COMPASS [8] to extract a first transversity distribution for up and down quarks in the nucleon [9].

\section{4. - Interference fragmentation}

The second chiral-odd fragmentation function available to access transversity is the interference fragmentation function. The interference fragmentation function relates the transverse quark spin to an azimuthal distribution of a hadron pair around the quarks axis. Because of measuring hadron pairs, one does not rely on transverse momentum relative to the reference axis an thus conventional collinear evolution applies which is well known. In the Belle a pair of pions with opposite charge is selected in each hemi- 


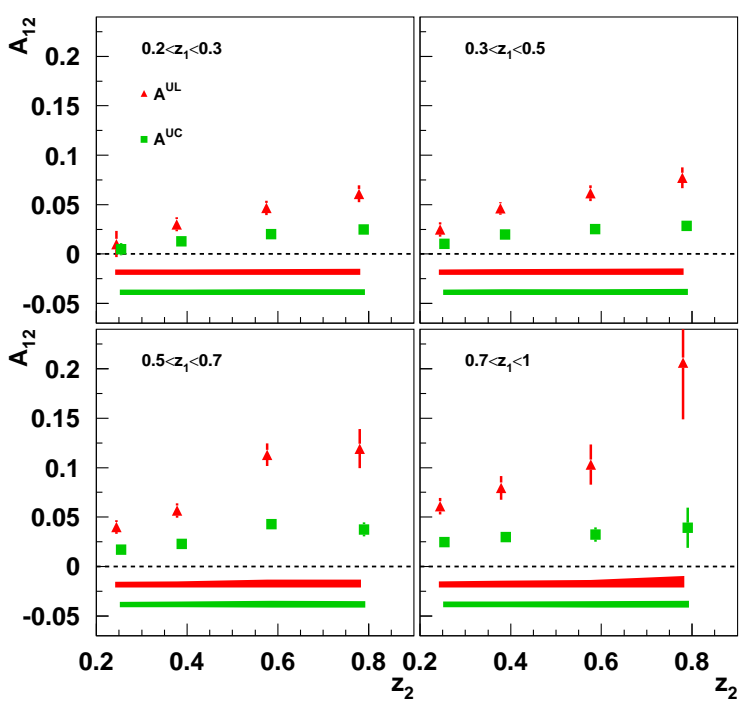

Fig. 1. - Cosine modulation $A_{12}$ proportional to the Collins functions in oppositely charged pion pairs over like sign pion pairs (red) as well as over all pion pairs (green) as a function of the fractional energie of both pions.

sphere [10]. Apart from the same selection criteria as for the Collins analysis an opening angular cut relative to the thrust axis is applied which removes acceptance effects and the normalized yields could directly be fit with the corresponding cosine modulation which is proportional to the product of IFFs for the quark and antiquark side. Belle has successfully measured these asymmetries as a function of the fractional energies of the two pairs as well as its invariant masses and sizeable asymmetries were found [11]. They are increasing with increasing fractional energy and are also increasing up to invariant masses of about $0.8 \mathrm{GeV}$ before they seem to level off as can be seen in fig. 2 .
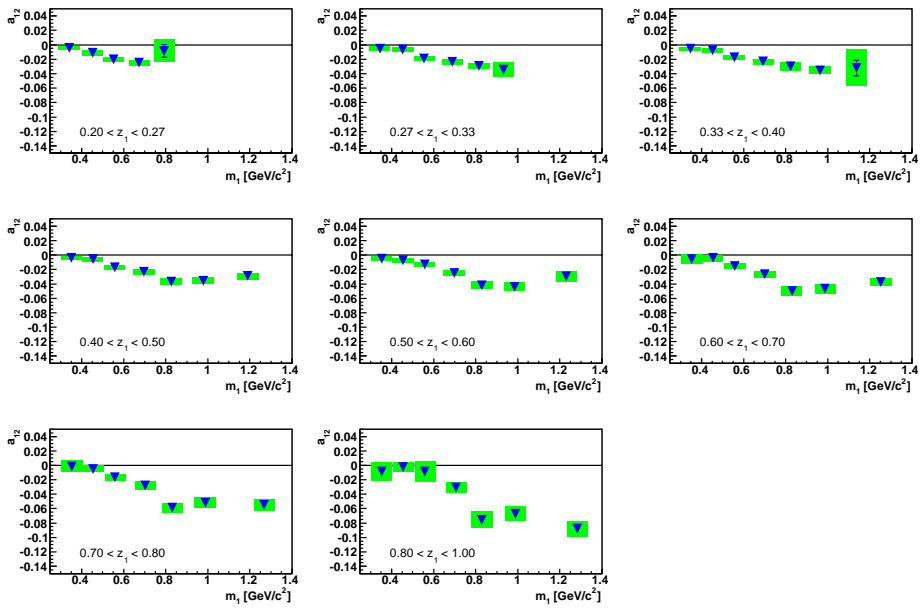

Fig. 2. - Cosine modulation proportional to the interference fragmentation fucntion in pairs of oppositely charged pion pairs as a function of one pairs fractional energy and invariant mass. 
Together with the SIDIS IFF measurements from HERMES [12] and recently COMPASS [13] the Pavia group extracted the valence transversity distributions in a collinear frameworks [14]. The preliminary results at RHIC $[15,16]$ have not yet been included.

\section{5. - Outlook}

While the first direct measurements of the Collins and interference fragmentation functions made the access to the transversity distribution function possible, several limitations remain. The most importatnt limitation is, that so far only pions or pion pairs have been studied. This implies, that one is mostly sensitive to the up and down valence quarks in transversity related SIDIS or pp measurements. Including kaons and pionkaon combinations will allow a more sophisitcated flavor decomposition of transversity. Furthermore HERMES has measured also kaon Collins asymmetries already [17] so the measurement of the related kaon Collins fragmentation function is needed. The analysis of the kaon related Collins and interference fragmenation function extraction is ongoing and first results are expected soon. Also the asymmetries for Vector mesons are being studied. Another important aspect of the Collins fragmentation function is, that the transverse momentum dependence has not been studied too well and the transvsersity extractions generally assume a Gaussian dependence. A detailed measurement of the transverse momentum dependence would significantly improve the understanding of not only the Collins function itself but also that of other transverse momentum dependent distribution functions. Initial studies have been performed for transverse momenta related to the thrust axis but acceptance effects and transverse momentum smearing need to be furhter investigated before results can be expected.

\section{REFERENCES}

[1] Abashian A. et al. (Belle Collaboration), Nucl. Instrum. Methods A, 479 (2002) 117.

[2] Kurokawa S. and Kikutani E., Nucl. Instrum. Methods A, 499 (2003) 1, and other papers in these proceedings.

[3] Collins J. C., Heppelmann S. F. and Ladinsky G. A., Nucl. Phys. B, 420 (1994) 565.

[4] Bravar A. et al. (Fermilab E704 Collaboration), Phys. Rev. Lett., 77 (1996) 2626.

[5] Seidl R. et al. (Belle Collaboration), Phys. Rev. Lett., 96 (2006) 232002.

[6] Seidl R. et al. (Belle Collaboration), Phys. Rev. D, 78 (2008) 032011; 86 (2012) 039905(E).

[7] Airapetian A. et al. (Hermes Collaboration), Phys. Rev. Lett., 94 (2005) 012002.

[8] Alexakhin V. Y. et al. (COMPASS Collaboration), Phys. Rev. Lett., 94 (2005) 202002.

[9] Anselmino M., Boglione M., D’Alesio U., Kotzinian A., Murgia F., Prokudin A. and Turk C., Phys. Rev. D, 75 (2007) 054032.

[10] Boer D., Jakob R. and Radici M., Phys. Rev. D, 67 (2003) 094003.

[11] Vossen A. et al. (Belle Collaboration), Phys. Rev. Lett., 107 (2011) 072004.

[12] Airapetian A. et al. (HERMES Collaboration), JHEP, 0806 (2008) 017.

[13] Adolph C. et al. (COMPASS Collaboration), Phys. Lett. B, 713 (2012) 10.

[14] Bacchetta A., Courtoy A. and Radici M., JHEP, 03 (2013) 119.

[15] Yang R. (PheniX Collaboration), AIP. Conf. Proc., (2009) 1182.

[16] Vossen A. (STAR Collaboration), talk presented at RHIC/AGS users meeting 2012

[17] Airapetian A. et al. (Hermes Collaboration), Phys. Lett. B, 693 (2010) 11. 